\title{
Enhancing Hydrogen Diffusion in Silica Matrix by Using Metal Ion Implantation to Improve the Emission Properties of Silicon Nanocrystals
}

\author{
J. Bornacelli, J. A. Reyes-Esqueda, L. Rodríguez-Fernández, J. L. Ruvalcaba-Sil, \\ F. J. Jaimes, and A. Oliver
}

Instituto de Física, Universidad Nacional Autónoma de México, 04510 México, DF, Mexico

Correspondence should be addressed to A. Oliver; oliver@fisica.unam.mx

Received 18 September 2013; Accepted 28 November 2013; Published 6 January 2014

Academic Editor: O. K. Tan

Copyright (c) $2014 \mathrm{~J}$. Bornacelli et al. This is an open access article distributed under the Creative Commons Attribution License, which permits unrestricted use, distribution, and reproduction in any medium, provided the original work is properly cited.

\begin{abstract}
Efficient silicon-based light emitters continue to be a challenge. A great effort has been made in photonics to modify silicon in order to enhance its light emission properties. In this aspect silicon nanocrystals (Si-NCs) have become the main building block of silicon photonic (modulators, waveguide, source, and detectors). In this work, we present an approach based on implantation of Ag (or Au) ions and a proper thermal annealing in order to improve the photoluminescence (PL) emission of Si-NCs embedded in $\mathrm{SiO}_{2}$. The $\mathrm{Si}-\mathrm{NCs}$ are obtained by ion implantation at $\mathrm{MeV}$ energy and nucleated at high depth into the silica matrix (1-2 $\mu \mathrm{m}$ under surface). Once $\mathrm{Si}$-NCs are formed inside the $\mathrm{SiO}_{2}$ we implant metal ions at energies that do not damage the Si-NCs. We have observed by, $\mathrm{PL}$ and time-resolved PL, that ion metal implantation and a subsequent thermal annealing in a hydrogen-containing atmosphere could significantly increase the emission properties of Si-NCs. Elastic Recoil Detection measurements show that the samples with an enhanced luminescence emission present a higher hydrogen concentration. This suggests that ion metal implantation enhances the hydrogen diffusion into silica matrix allowing a better passivation of surface defects on Si NCs.
\end{abstract}

\section{Introduction}

Despite the great effort made in silicon photonics to achieve efficient Silicon Nanocrystals (Si-NCs), light emitters embedded in a solid silicon dioxide $\left(\mathrm{SiO}_{2}\right)$ matrix, the photoluminescence (PL) from that kind of devices continues to be too weak for many practical applications [1]. The reason for that resides in two principal aspects: one of them is the wellknown quasi-direct band gap in silicon nanocrystals which reduces the probability for radiative transition [1-3], and the other is related to the fact that PL properties of Si-NCs are strongly affected by their surface defects $[4,5]$. The Pb defects, or dangling bonds, are known to cause quenching of the PL from Si-NCs [4-6]. Much research has been done to find a method to enhance the PL emission from a system of Si-NCs in $\mathrm{SiO}_{2}$ by controlling the passivation of their surface defects by using gases such as hydrogen $\left(\mathrm{H}_{2}\right)$, nitrogen $\left(\mathrm{N}_{2}\right)$, or oxygen $\left(\mathrm{O}_{2}\right)$ [7-14]. It is well known that a thermal treatment in a molecular hydrogen-containing atmosphere increases the PL from Si-NCs more than Nitrogen or Oxygen [7].
This could be related to the fact that hydrogen diffusion in silica is higher, and consequently a great number of surface defects can be passivated. In many pieces of work the SiNCs are formed by ion implantation at energies between 35 and $400 \mathrm{KeV}$ [4, 5, 7-11]. At those energies the Si-NCs are formed near the surface at distances not exceeding $1 \mu \mathrm{m}$. This allows a great quantity of hydrogen to diffuse until it reaches the region where the Si-NCs are nucleated but at the expense of robustness and protection from external damage. Another method to increase the PL of Si-NCs even more is known as Alneal [10] and it consists in depositing a thin film of $\mathrm{Al}$ onto the surface of $\mathrm{SiO}_{2}$, where the $\mathrm{Si}-\mathrm{NCs}$ were previously formed. Then, when a thermal treatment in hydrogen is done, the Al film allows the dissociation of the molecular hydrogen. This atomic hydrogen greatly enhances the PL in contrast to that obtained under thermal treatment in molecular hydrogen. Therefore, diffusion of hydrogen in silica matrix is of great importance to achieve a better control over the passivation of surface defects on Si-NCs and, 
therefore, their PL properties. In this work, we have used metal $(\mathrm{Ag}$ or $\mathrm{Au})$ ion implantation in order to increase the diffusion and solubility of hydrogen in a silica matrix where $\mathrm{Si}-\mathrm{NCs}$ were previously formed by ion implantation. As a result, the surface defects on Si-NCs can be better passivated and improve their PL properties.

The Si-NCs synthesis has been done by ion implantation at $\mathrm{MeV}$ energies. So the Si-NCs are nucleated at a depth between 1 and $2 \mu \mathrm{m}$. At those distances from the surface, the Si-NCs are better protected from mechanical or chemical damage which is desirable for microelectronic applications. Once the Si-NCs are formed inside the silica matrix, the metal ions are implanted at energies that do not overlap with the previously implanted Si ions. Finally, a thermal treatment in hydrogen-containing atmospheres is done. As we will later show, this method allows us to increase the PL emission from $\mathrm{Si}-\mathrm{NCs}$, as a consequence of enhancing hydrogen diffusion until it reaches the region where the Si-NCs were formed.

\section{Experimental Details}

Silicon nanocrystals synthesis has been done by the nucleation of silicon precipitates in a high-purity silica matrix (Suprasil 300). The excess of silicon is introduced by ion implantation at room temperature with $1.5 \mathrm{MeV} \mathrm{Si}^{+2}$ ions at a fluence of $2.5 \times 10^{17} \mathrm{ions} / \mathrm{cm}^{2}$, using the $3 \mathrm{MV}$ Tandem accelerator (NEC 9SDH-2 Pelletron) facility at the Instituto de Física of the Universidad Nacional Autónoma de México (IFUNAM). The SRIM code was applied to calculate the concentration profile of the Si-implanted ions $[15,16]$. Previously, two methods to nucleate the Si-NCs applying thermal annealing were studied [13]. One procedure consisted in onestep annealing process at $1100^{\circ} \mathrm{C}$ for $1 \mathrm{hr}$ under a reducing atmosphere (RA) of $50 \% \mathrm{~N}_{2}$ and $50 \% \mathrm{H}_{2}$. The second one consisted in two-step annealing: the first thermal treatment was done at $1100^{\circ} \mathrm{C}$ for $1 \mathrm{hr}$ under a $100 \%$ Ar atmosphere, and the application of a second one took place at $510^{\circ} \mathrm{C}$ under RA by $1 \mathrm{hr}$. This two-step process is currently used in other pieces of work and is suggested to be the best method to synthesize Si-NCs in a silica matrix $[7,10,11]$. The samples produced in this work are denoted as $\mathrm{A}$ and $\mathrm{B}$, depending on the one- and two-step methods applied to nucleate the SiNCs, respectively.

Some samples with Si-NCs formed inside the silica matrix by the one-step method were implanted with $1 \mathrm{MeV} \mathrm{Ag}^{+2}$ ions and others with $1.9 \mathrm{MeV} \mathrm{Au}{ }^{+2}$ at a fluence of $6 \times$ $10^{16}$ ions $/ \mathrm{cm}^{2}$ in both cases. These implantation energies were chosen in order to have $\mathrm{Ag}$ and $\mathrm{Au}$ ion distributions closer to the surface and to avoid the spatial overlapping with the previously created Si-NCs distributions, thus minimizing the damage over them. The ion ranges of the $\mathrm{Ag}$ and $\mathrm{Au}$ implantations were corroborated by means of Rutherford Backscattering Spectrometry (RBS) [17], using a $2 \mathrm{MeV}^{4} \mathrm{He}^{++}$ beam. Subsequently, the samples $\mathrm{A}(\mathrm{Ag})$ and $\mathrm{A}(\mathrm{Au})$ were thermally annealed under RA at $600^{\circ} \mathrm{C}$. At this temperature, the sample with $\mathrm{Ag}$ ion implantation formed Ag-NPs [25]. On the other hand, a piece of a sample A ( $\mathrm{Au})$ was thermally annealed under oxidant atmosphere (OA), consisting of air at $1100^{\circ} \mathrm{C}$ for $1 \mathrm{hr}$ (sample A (Au)-1) in order to nucleate Au-NPs inside the silica matrix [18].

The hydrogen depth profile of all samples was measured using Elastic Recoil Detection (ERD) [19] of a $8.5 \mathrm{MeV}^{4} \mathrm{He}^{++}$ ion beam incident at $75^{\circ}$ from the normal surface. Recoiled Hydrogen was detected at a scattering angle of $30^{\circ}$. In front of the detector window a $80 \mu \mathrm{m}$ Mylar foil was placed to stop scattered He ions. Simultaneously, RBS measurements at $168^{\circ}$ were carried out in order to quantify the integrated charge deposited on the sample during the ERD measurements. The depth profiles of hydrogen were deduced from the energy spectra using the SIMNRA simulation code $[20,21]$. To simulate the ERD spectra, the profile concentration of $\mathrm{Ag}$ and $\mathrm{Au}$ measured by RBS and the calculated Si concentration depth profile were taken into account for sample composition.

Photoluminescence (PL) measurements were performed at room temperature at an excitation wavelength of $355 \mathrm{~nm}$ using ps pulses, at a frequency repetition rate of $10 \mathrm{~Hz}$, from a laser system PL2143A by EKSPLA. PL signal was collected by a 1000-micron optical fiber and detected by an Ocean Optics USB2000+ spectrometer. The time-resolved PL was measured using a Hamamatsu H10721-20 photomultiplier module coupled to an Acton Series SP2300 monochromator. The PL signal was visualized on a digital oscilloscope and was resolved in a microsecond scale. Emission decay lifetimes were extracted by the least-square fitting of a stretched exponential [22,23]. The optical absorption of all the samples was measured by means of a Varian Cary 5000 UV-VIS spectrophotometer. The Si-NCs were observed by using Transmission Electron Microscopy with a JEOL 2010F. To do this, the sample was devastated mechanically in the opposite side of the ion implantation and in the normal direction to the surface of the sample up to $20 \mu \mathrm{m}$, and then a Precision Ion Polishing System (PIPS) was used to devastate the sample in the side of the implantation up to a few hundred nanometers.

\section{Results and Discussion}

The Transmission Electron Microscopy (TEM) images in Figure 1 confirm the formation of Si-NCs inside the silica matrix. A large quantity of small-sized Si-NCs $(\sim 3-5 \mathrm{~nm}$ of diameter) can be visualized in the images. However, it is also possible to observe some NCs greater than $10 \mathrm{~nm}$ of diameter (as shown in Figure 1(b)). Bigger Si-NCs could be formed inside the $\mathrm{SiO}_{2}$ as a result of an excessive fluence $\mathrm{Si}$ ion implantation.

Figure 2 shows the typical PL spectra of the sample with $\mathrm{Si}$-NCs embedded in a silica matrix. The continuous line corresponds to A samples while dashed line to B samples. From these results, the one-step annealing procedure was clearly the best method to achieve a higher PL emission intensity.

Figure 3 shows the hydrogen depth profile measured by ERD; the open triangles correspond to A samples and open squares to $\mathrm{B}$ samples. Also the $\mathrm{Si}$ ions projected range calculated by the SRIM code is shown in this figure (open rhombs) to compare with hydrogen distributions. The calculated $\mathrm{Si}$ ion implanted distribution has a broad depth 


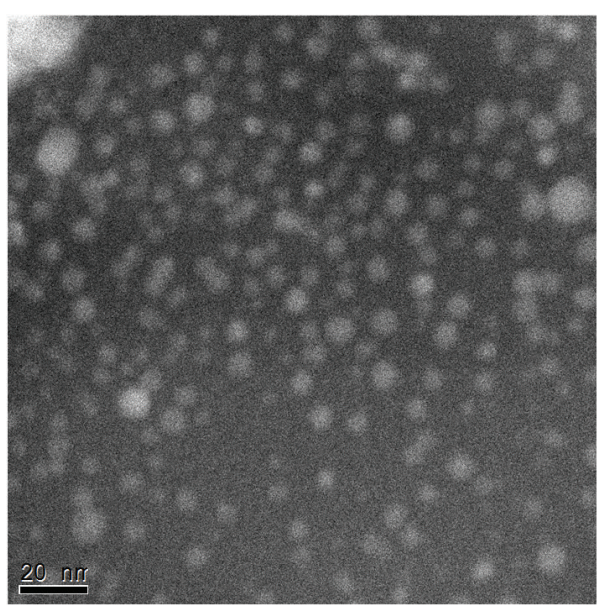

(a)

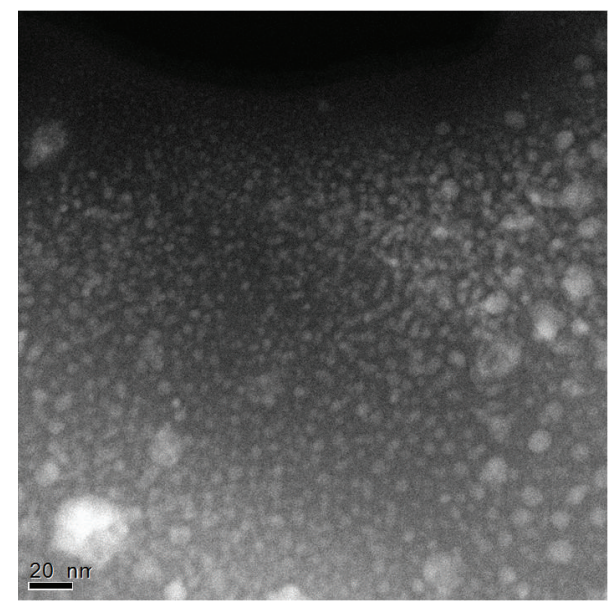

(b)

FIGURE 1: Plan-view TEM micrograph, obtained by using Z contrast technique (HAADF), showing Si-NCs formed inside a silica matrix $\left(\mathrm{SiO}_{2}\right)$ after being annealed at $1100^{\circ} \mathrm{C}$ in $\mathrm{RA}$ a piece of $\mathrm{SiO}_{2}$ with $\mathrm{Si}$ ions implantation $\left(1.5 \mathrm{MeV}\right.$ at a fluence of $2.5 \times 10^{17}$ ions $\left./ \mathrm{cm}^{2}\right)$.

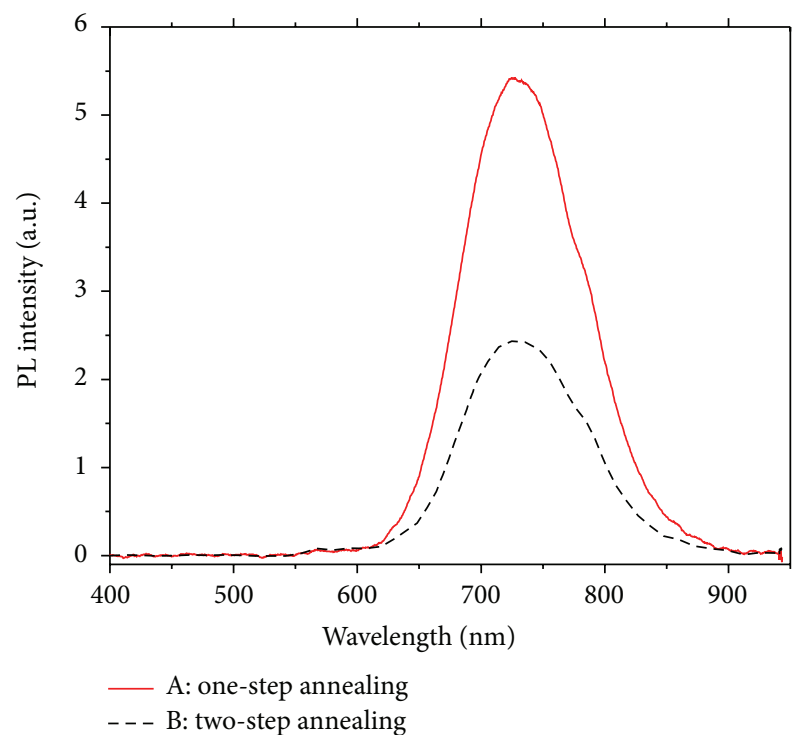

FIGURE 2: PL emission from Si-NCs embedded in silica matrix and prepared by two methods as described above: A (red curve) and B (black dashed curve).

distribution of at least $1 \mu \mathrm{m}$, ranging from 1 to $2 \mu \mathrm{m}$ under silica surface. For both types of samples a large amount of hydrogen concentration is observed near the silica surface and decreases as we move into the sample. From $\sim 0.5$ to $2.2 \mu \mathrm{m}$, it is possible to observe clearly an accumulation of hydrogen that overlaps with the Si-implanted depth profile calculated by using the SRIM program. This result has been observed before in samples with embedded Si-NCs and was explained considering that at the interface of $\mathrm{SiO}_{2} / \mathrm{Si} \mathrm{Si}$ NCs can easily trap hydrogen, where it passivates dangling bonds [14]. The Si-NCs synthesized by method A present a greater accumulation of hydrogen than the sample prepared by method $\mathrm{B}$. This result is related to the silicon ion implantation-induced damage. So when the sample is heated

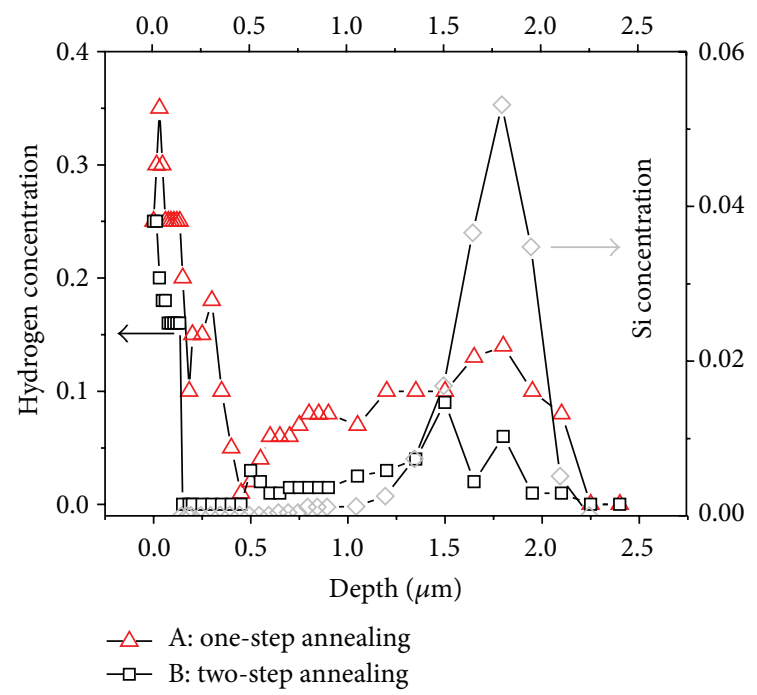

FIGURE 3: Hydrogen depth profile measured by ERD. Open triangles and squares correspond to the hydrogen concentration for the samples A and B, respectively. The silicon implanted concentration (gray rhombs) calculated by SRIM is shown for comparison.

in $\mathrm{RA}$ at $1100^{\circ} \mathrm{C}$, the diffusion of hydrogen into silica matrix is enhanced by the presence of this damage. The hydrogen concentration in silica glass depends on the number of available sites in the silica structure to dissolve the hydrogen atoms [24]. The silicon implantation creates vacancies in the silica matrix, which directly increase the number of sites available to dissolve hydrogen. As a result, a great quantity of hydrogen could reach the region where the Si-NCs were formed. This effect could not take place in sample B because it is initially heated under Argon gas at $1100^{\circ} \mathrm{C}$, allowing the matrix recovery. When this sample is heated again under RA, to passivate surface defects on Si-NCs, the matrix has been restored and the amount of vacancies is much lower, reducing the diffusion of hydrogen into the silica. 
On the other hand, the hydrogen distribution is broader than the theoretical Si-implanted depth profile, and a remarkable hydrogen concentration appears between 0.5 and $1.5 \mu \mathrm{m}$, where the excess of Si implanted is smaller. As was pointed out before [14], this could be explained considering that the amount of retained hydrogen is proportional to the surface area of the nanocrystals. Smaller nanocrystals are probably formed at a region where the excess of implanted $\mathrm{Si}$ is smaller. SRIM calculations show an asymmetric Si distribution, and we expect that a great number of smaller Si-NCs could be nucleated at depths less than $1.5 \mu \mathrm{m}$. So even though at a greater depth a large quantity of Si implanted is present, most of these ions contribute to the volume of large nanocrystals, and the surface available to trap hydrogen decreases. In contrast, smaller nanocrystals have a greater surface-volume ratio; that is, most of implanted Si are forming the nanocrystal surface and can trap more hydrogen [14].

If the silicon ion implantation was made at lower energies $(\mathrm{keV})$, as it is frequently done in other works [4, 5, 7-11], the $\mathrm{Si}-\mathrm{NCs}$ would be formed near the surface of the silica matrix. At these depths there is a high hydrogen concentration, as we can see in Figure 3, and the PL emission from Si-NCs nucleated in that region could be more significant. However, the Si-NCs would be closer to the sample surface and more exposed to external physical and chemical damage, and it makes the systems of Si-NCs optically unstable. As we will show, another way to improve the emission properties of $\mathrm{Si}-\mathrm{NCs}$ is to implant metal ions like Ag or Au. Figure 4(a) shows the PL emission of sample after Ag implantation and a subsequent thermal heating in RA at $600^{\circ} \mathrm{C}$ (sample A (Ag) red line). The dashed-doted black line is the reference (sample A).

The PL intensity from sample A (Ag) is approximately twofold enhanced. The spectrum is also slightly blue-shifted $(\sim 10 \mathrm{~nm})$. The inset graph in Figure 4(a) shows the typical absorption band of Ag-NPs formed in sample A (Ag) after thermal treatment at $600^{\circ} \mathrm{C}$. The absorption band is directly related to the electrons plasma oscillation in the Ag-NPs, and it is well known as Surface Plasmon Resonance (SPR) [25-27]. And the maximum value of the absorption band corresponds to the wavelength excitation light to which the electrons plasma oscillation inside the Ag-NPs is greater. The presence of Ag-NPs could explain the enhanced PL emission by a possible plasmonic interaction with Si-NCs. However, the required metal NPs size (currently $>10 \mathrm{~nm}$ ) and the distance needed to observe this effect ( 20 to $50 \mathrm{~nm})$ [26-28] make this effect unlikely in our system. Table inset in Figure 4(a) shows the PL lifetime measured value of $\tau$ and stretching parameter $\beta$. As it is shown there, PL lifetime has increased in the sample with metal ion implantation. As mentioned in other works, this could be related to improving surface defects passivation on Si-NCs [4, 7, 10-13]. Hydrogen depth profile of the sample with Ag implantation and its reference without it are shown in Figure 4(b). Black circles show the measured Ag depth profile by RBS, and open gray rhombs are the theoretical Si depth profile by SRIM simulation. As we can see there, the diffused hydrogen increases into the sample with Ag ion implantation. Hydrogen concentration in the range where the $\mathrm{Ag}$ is present is similar to that observed in the reference sample at its surface. On the other hand, the hydrogen concentration increases, nonuniformly, in the range where Si ions were implanted. Where we expected a large quantity of smaller nanocrystals (distance less than $1.5 \mu \mathrm{m}$ ), we observe a greater hydrogen concentration. That means that a large number of smaller Si-NCs have been passivated with hydrogen, and probably most of them, whose surface defects had inhibited then emission properties, now could be activated to emit light. Smaller nanocrystals emit light at shorter wavelengths and this explains why the PL spectrum of the sample with Ag ion implantation is blueshifted [2, 3, 5, 7-9].

A similar result can be observed in a sample with Si-NCs that has been implanted with Au ions. Figure 5(a) shows the PL spectrum of a sample implanted with Au ions and thermal annealing at $1100^{\circ} \mathrm{C}$ (sample $\mathrm{A}(\mathrm{Au})-1$ ) or $600^{\circ} \mathrm{C}$ (sample A (Au)-2) under OA. A third sample was heated at $600^{\circ} \mathrm{C}$ under RA (sample A $(\mathrm{Au})$ ). PL emission from the reference sample, that is, without $\mathrm{Au}$ ion implantation (sample $\mathrm{A}$ ), is also shown. As we can see, the PL enhancement factor is greater in sample $\mathrm{A}(\mathrm{Au})$. On the other hand, sample $\mathrm{A}(\mathrm{Au})-2$ has a quenching of PL intensity, probably because the Au ion implantation has damaged some Si-NCs. The inset graph in Figure 5(a) shows the absorption spectrum of each sample. Sample A ( $\mathrm{Au})-1$ has a defined absorption band (dasheddoted blue curve in the inset) due to SPR of Au-NPs [18] but has a smaller increment of PL emission compared with sample A ( $\mathrm{Au}$ ), which does not have Au-NPs formed (doted red curve in the inset). In consequence, we can deduce that the presence of Au-NPs does not have any appreciable effect over the final PL emission.

Inset table in Figure 5(a) shows PL lifetime measurements for the set samples A, A ( $\mathrm{Au})$, and A (Au)-1. These results show that PL lifetime $(\tau)$ is much greater for the sample with a greater PL intensity, which is a consequence of a better surface defects passivation of their Si-NCs. Figure 5(b) shows hydrogen depth profiles of the samples with Au implantation. Gray circles show Au depth profile obtained by RBS measurements. According to the results in Figure 5(b), sample A (Au)-1 has more or less the same hydrogen concentration in the region where Si ions were implanted (open gray rhomb) compared with the reference sample A. However, as we can see in Figure 5(a), the PL emission from sample A (Au)-1 is slightly enhanced. We think this PL increase could have two contributions. One of them is related to the fact that the sample $\mathrm{A}(\mathrm{Au})-1$ was heated twice at $1100^{\circ} \mathrm{C}$, the temperature at which new Si-NCs could be formed, and those who have been damaged by previous Au ion implantation can be nucleated again. And the second one is related to the presence of nitrogen in the air $(\sim 70 \%)$, which was the annealing atmosphere for this sample. The nitrogen can diffuse into silica at high temperature $\left(\sim 1000^{\circ} \mathrm{C}\right)[29,30]$, and it is well known that nitrogen can passivate surface defects on Si-NCs, leading to an increase in their emission properties $[7,8]$. On the other hand, hydrogen concentration in sample $\mathrm{A}(\mathrm{Au})$, heated at $600^{\circ} \mathrm{C}$ in $\mathrm{RA}$, has a significant increase, greater than that observed for sample A (Au)-1. Moreover, sample A (Au) has a hydrogen concentration greater than that obtained with Ag ion implantation (see Figures 4(b) and 5(b)). 


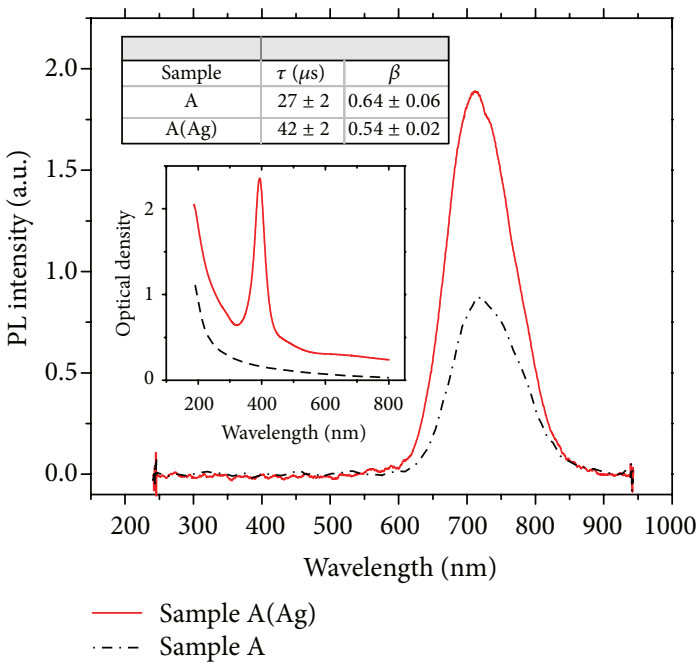

(a)

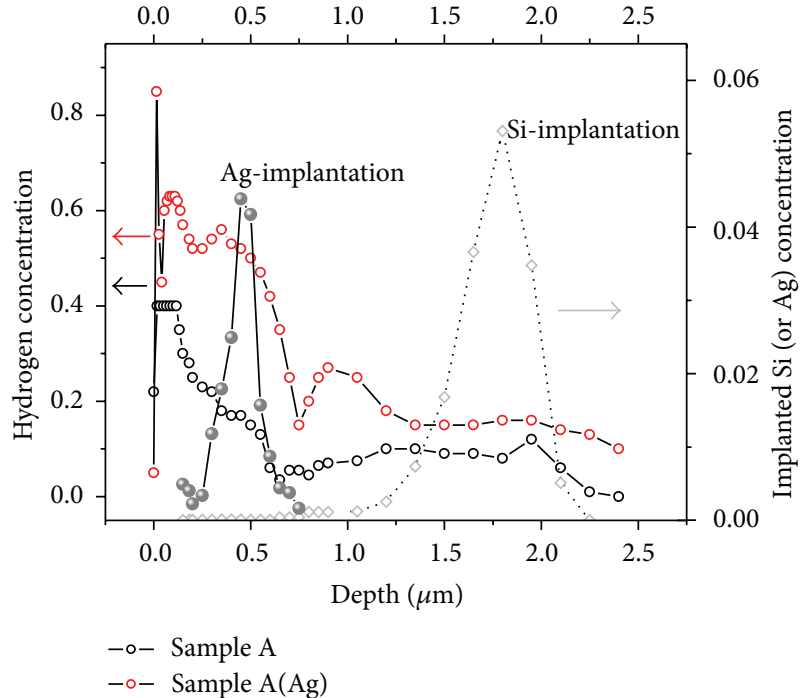

(b)

Figure 4: (a) PL emission from samples A (dashed black curve) and A (Ag) (red line). The inset graph shows the respective optical density. Inset table shows the parameters fitting of the measured PL decay curve of each sample according to the stretched exponential model mentioned above. (b) Hydrogen depth profile by ERD. Black triangles and red circles show the hydrogen concentration of samples A and A (Ag), respectively. Ag depth profile (filled gray circle) by RBS and Si depth profile (open gray rhomb) by SRIM simulation are shown for comparison.

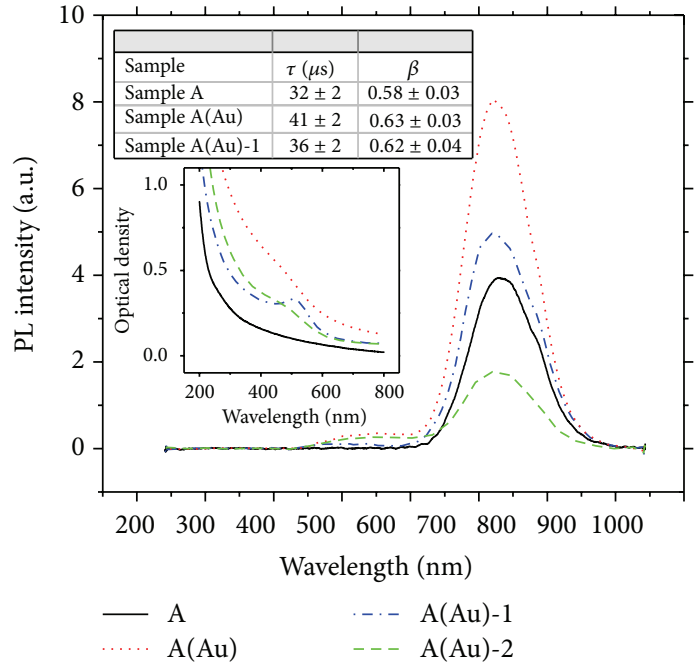

(a)

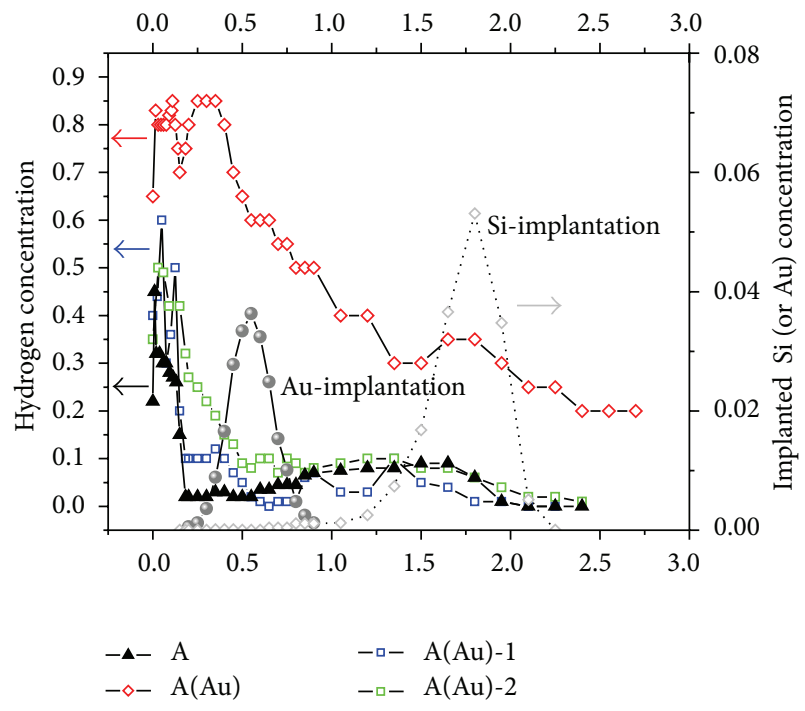

(b)

Figure 5: (a) PL emission from samples $\mathrm{A}, \mathrm{A}(\mathrm{Au})$, and $\mathrm{A}(\mathrm{Au})-1$. Inset graph shows the absorption for each sample. PL lifetime parameters are shown in the inset table. (b) Hydrogen depth profile of the samples A, A ( $\mathrm{Au}$ ), and A (Au)-1. Au depth profile (filled gray circle) by RBS and Si depth profile (open gray rhomb) by SRIM simulation are shown for comparison.

That is because $\mathrm{Au}$ ions are much more massive than $\mathrm{Ag}$ ions. In consequence, $\mathrm{Au}$ ions can produce more vacancies and the diffusion and solubility of hydrogen inside the silica matrix could increase even more. Despite greater hydrogen diffusion in the sample with $\mathrm{Au}$ implantation (sample $\mathrm{A}(\mathrm{Au})$ ), the increase of its PL emission is of the same level as that observed in the sample with $\mathrm{Ag}$ ion implantation (sample $\mathrm{A}(\mathrm{Ag})$ ). This could be explained taking into account that, given a system of Si-NCs, there is a finite number of surface defects on them that could be passivated with hydrogen; in consequence their PL improvement must have a maximum that is achievable by surface defects passivation.

Improving PL emission from Si-NCs by using metal ion implantation allows us to control the magnitude of their final PL intensity. In Figure 6(a), a reduction of the PL intensity in a sample with Si-NCs (sample A) after being heated for 


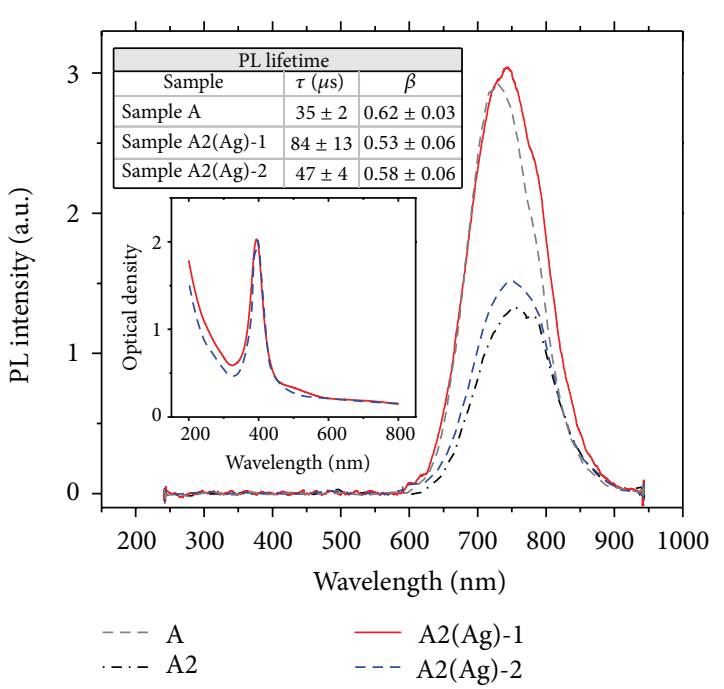

(a)

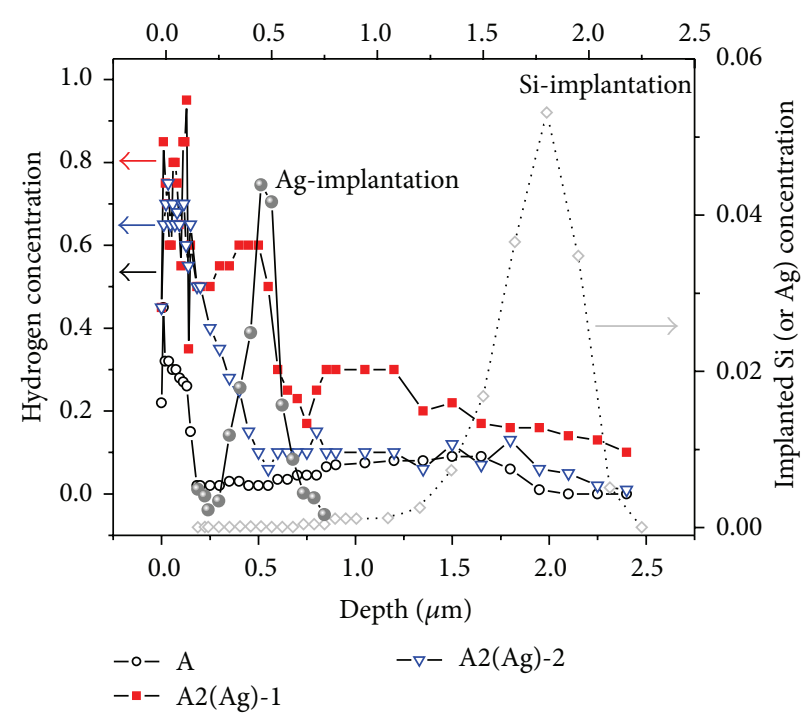

(b)

Figure 6: (a) PL emission from samples A, A2, A2 (Ag)-1, and A2 (Ag)-2. Inset graph shows the optical absorption of each sample. PL lifetime fitting parameters are shown in the inset table. (b) Hydrogen depth profile of each sample, Ag depth profile (filled gray circles) by RBS, and Si depth profile (open gray rhombs) by SRIM simulation are shown for comparison.

$2 \mathrm{~h}$, at $1100^{\circ} \mathrm{C}$ in $\mathrm{RA}$, is shown (sample $\mathrm{A} 2$ ). PL spectrum is also red-shifted. After this second thermal heating, the average size of Si-NCs increases and, as a consequence of less quantum confinement, their PL emission is red-shifted, and their efficiency is reduced [2, 3]. Then sample A2 was implanted with $\mathrm{Ag}$ ions at the same conditions explained before and cut into two pieces. One piece was heated at $600^{\circ} \mathrm{C}$ for $1 \mathrm{~h}$ under RA (sample A2 (Ag)-1) and the other under Argon gas (sample A2 (Ag)-2).

As we can see in Figure 6(a), although both samples have an absorption band near $400 \mathrm{~nm}$, due to the SPR of Ag-NPs formed inside the silica matrix, it is the presence of hydrogen in the annealing atmosphere that remarkably affects the final PL emission of sample A2 (Ag)-1. On the other hand, the small PL increment observed in sample A2 (Ag) -2 could be explained as a result of additional surface defects passivation by means of the hydrogen previously dissolved in the silica matrix. Hydrogen depth profiles in Figure 6(b) show that hydrogen concentration of sample A2 (Ag)-2, in the region where the Si-NCs were formed, is almost similar to that in the reference sample A. Taking into account that Ag-NPs in sample A2 (Ag)-2 were nucleated under argon gas annealing atmosphere, that is, no hydrogen was present, we can deduce that what we can observe from the hydrogen depth profile of this sample is the same for sample A2. This means that the decrease of PL intensity from sample A2 mainly comes from an increasing mean size of their Si-NCs, rather than hydrogen desorption [11]. Longer annealing times can nucleate more new and smaller Si-NCs and also produce bigger ones. The smaller and bigger nanocrystals could have more surface defects that reduce their PL emission (sample A2). This explains why sample A2 (Ag)-2 presents a significant increase of their PL emission when it is heated under RA.
And, as we can see in Figure 6(b), sample A2 (Ag)-1 has a higher hydrogen concentration. This means that a greater amount of hydrogen is trapped in the $\mathrm{SiO}_{2} / \mathrm{Si}$ interface. Surface defects on Si-NCs are passivated and then their PL emission is enhanced.

\section{Conclusions}

Si-NCs embedded in silica matrix at high depth (1-2 $\mu \mathrm{m})$ were synthesized by ion beam implantation and subsequent thermal treatments. The procedure of applying only one annealing in $\mathrm{RA}$ at $1100^{\circ} \mathrm{C}$ generates higher PL because it allows a better passivation of surface defects on Si-NCs since the silicon ion implantation damage allows a higher hydrogen diffusion into the silica matrix. Also, we show that metal ion implantation is an efficient way to enhance the solubility and diffusion of molecular hydrogen into the silica samples. This allows improving the passivation process of surface defects on Si-NCs and, in consequence, their PL emission is twofold increased. The hydrogen is trapped in regions that coincide with the range where the Si ions implantation is distributed. Enhancing solubility and hydrogen diffusion in silica also allow us a better control over the final PL intensity of a system of Si-NCs embedded in it. We observed that the PL intensity of Si-NCs decreases and their spectrum is red-shifted, when the sample is overheated for more than two hours. However, the PL intensity is recovered when we implanted Ag ions and the sample is again thermally treated in a hydrogencontaining atmosphere. Even though for long annealing time we obtain bigger and photoluminescent less-efficient Si-NCs, we can improve their PL emission by inducing damage in the silica matrix by means of metal ion implantation and finally by heating the sample in a hydrogen-containing atmosphere. 


\section{Conflict of Interests}

The authors declare that there is no conflict of interests regarding the publication of this paper.

\section{Acknowledgments}

The authors wish to acknowledge the technical assistance of K. López and J. G. Morales. They also acknowledge financial support from PAPIIT-UNAM through Grants IN108412 and IT102013, from CONACyT through Grant 102937, and from AFOSR through Grant FA9550-12-1-0235.

\section{References}

[1] N. Daldosso and L. Pavesi, "Nanosilicon photonics," Laser and Photonics Reviews, vol. 3, no. 6, pp. 508-534, 2009.

[2] D. Kovalev, H. Heckler, G. Polisski, and F. Koch, "Optical properties of Si nanocrystals," Physica Status Solidi (B), vol. 215, no. 2, pp. 871-932, 1999.

[3] V. A. Belyakov, V. A. Burdov, R. Lockwood, and A. Meldrum, "Silicon nanocrystals: fundamental theory and implications for stimulated emission," Advances in Optical Technologies, vol. 2008, Article ID 279502, 32 pages, 2008.

[4] M. López, B. Garrido, C. García et al., "Elucidation of the surface passivation role on the photoluminescence emission yield of silicon nanocrystals embedded in $\mathrm{SiO}_{2}$," Applied Physics Letters, vol. 80, no. 9, pp. 1637-1639, 2002.

[5] B. G. Fernandez, M. López, C. García et al., "Influence of average size and interface passivation on the spectral emission of $\mathrm{Si}$ nanocrystals embedded in $\mathrm{SiO}_{2}$," Journal of Applied Physics, vol. 91, no. 2, pp. 798-807, 2002.

[6] D. Hiller, M. Jivanescu, A. Stesmans, and M. Zacharias, " $\mathrm{Pb}(0)$ centers at the Si-nanocrystal/ $\mathrm{SiO}_{2}$ interface as the dominant photoluminescence quenching defect," Journal of Applied Physics, vol. 107, no. 8, Article ID 084309, 2010.

[7] A. R. Wilkinson and R. G. Elliman, "The effect of annealing environment on the luminescence of silicon nanocrystals in silica," Journal of Applied Physics, vol. 96, no. 7, pp. 4018-4020, 2004.

[8] M. Bolduc, G. Genard, M. Yedji et al., "Influence of nitrogen on the growth and luminescence of silicon nanocrystals embedded in silica," Journal of Applied Physics, vol. 105, no. 1, Article ID 013108, 2009.

[9] M. L. Brongersma, A. Polman, K. S. Min, E. Boer, T. Tambo, and H. A. Atwater, "Tuning the emission wavelength of Si nanocrystals in $\mathrm{SiO}_{2}$ by oxidation," Applied Physics Letters, vol. 72, no. 20, pp. 2577-2579, 1998.

[10] A. R. Wilkinson and R. G. Elliman, "Passivation of Si nanocrystals in $\mathrm{SiO}_{2}$ : atomic versus molecular hydrogen," Applied Physics Letters, vol. 83, no. 26, pp. 5512-5514, 2003.

[11] A. R. Wilkinson and R. G. Elliman, "Kinetics of $\mathrm{H}_{2}$ passivation of Si nanocrystals in $\mathrm{SiO}_{2}$," Physical Review B, vol. 68, no. 15, Article ID 155302, pp. 1-8, 2003.

[12] R. Lockwood, S. McFarlane, J. R. Rodríguez Núñez, X. Y. Wang, J. G. C. Veinot, and A. Meldrum, "Photoactivation of silicon quantum dots," Journal of Luminescence, vol. 131, no. 7, pp. 15301535, 2011.
[13] J. Bornacelli, J. A. Reyes-Esqueda, L. Rodríguez-Fernández, and A. Oliver, "Improving passivation process of Si nanocrystals embedded in $\mathrm{SiO}_{2}$ using metal ion implantation," Journal of Nanotechnology, vol. 2013, Article ID 736478, 9 pages, 2013.

[14] S. P. Withrow, C. W. White, A. Meldrum, J. D. Budai, D. M. Hembree Jr., and J. C. Barbour, "Effects of hydrogen in the annealing environment on photoluminescence from Si nanoparticles in $\mathrm{SiO}_{2}$," Journal of Applied Physics, vol. 86, no. 1, pp. 396-401, 1999.

[15] Web page, http://www.srim.org/ .

[16] J. F. Ziegler, M. D. Ziegler, and J. P. Biersack, "SRIM-the stopping and range of ions in matter (2010)," Nuclear Instruments and Methods in Physics Research B, vol. 268, no. 11-12, pp. 1818-1823, 2010.

[17] J. A. Leavitt, L. C. Mclntyre Jr., and M. R. Weller, "Backscattering spectrometry," in Handbook of Modern Ion Beam Materials Analysis, J. R. Tesmer and M. Nastasi, Eds., Materials Research Society, Pittsburgh, Pa, USA, 1995.

[18] V. Rodriguez-Iglesias, O. Pena-Rodriguez, H. G. Silva-Pereyra et al., "Elongated gold nanoparticles obtained by ion implantation in Silica: characterization and T-matrix simulations," The Journal of Physical Chemistry C, vol. 114, no. 2, pp. 746-751, 2010.

[19] J. C. Barbour and B. L. Doyle, "Elastic recoil detection: ERD," in Handbook of Modern Ion Beam Materials Analysis, J. R. Tesmer and M. Nastasi, Eds., Materials Research Society, Pittsburgh, Pa, USA, 1995.

[20] M. Mayer, "SIMNRA User’s Guide," Technical Report IPP9/113, Max-Planck-Institut für Plasmaphysik, Garching, Germany, 1997.

[21] M. Mayer, "SIMNRA, a simulation program for the analysis of NRA, RBS and ERDA," in Proceedings of the 15th International Conference on the Application of Accelerators in Research and Industry, J. L. Duggan and I. L. Morgan, Eds., vol. 475, p. 541, American Institute of Physics Conference Proceedings, 1999.

[22] J. Linnros, N. Lalic, A. Galeckas, and V. Grivickas, "Analysis of the stretched exponential photoluminescence decay from nanometer-sized silicon crystals in $\mathrm{SiO}_{2}$," Journal of Applied Physics, vol. 86, no. 11, pp. 6128-6134, 1999.

[23] O. Guillois, N. Herlin-Boime, C. Reynaud, G. Ledoux, and F. Huisken, "Photoluminescence decay dynamics of noninteracting silicon nanocrystals," Journal of Applied Physics, vol. 95, no. 7, pp. 3677-3682, 2004.

[24] J. F. Shackelford, P. L. Studt, and R. M. Fulrath, "Solubility of gases in glass. II. He, $\mathrm{Ne}$, and $\mathrm{H}_{2}$ in fused silica," Journal of Applied Physics, vol. 43, no. 4, pp. 1619-1626, 1972.

[25] J. Roiz, A. Oliver, E. Muñoz, L. Rodríguez-Fernández, J. M. Hernández, and J. C. Cheang-Wong, "Modification of the optical properties of Ag-implanted silica by annealing in two different atmospheres," Journal of Applied Physics, vol. 95, no. 4, pp. 1783-1791, 2004.

[26] H. Mertens, A. F. Koenderink, and A. Polman, "Plasmonenhanced luminescence near noble-metal nanospheres: comparison of exact theory and an improved Gersten and Nitzan model," Physical Review B, vol. 76, no. 11, Article ID 115123, 2007.

[27] J. S. Biteen, L. A. Sweatlock, H. Mertens, N. S. Lewis, A. Polman, and H. A. Atwater, "Plasmon-enhanced photoluminescence of silicon quantum dots: simulation and experiment," Journal of Physical Chemistry C, vol. 111, no. 36, pp. 13372-13377, 2007. 
[28] J. S. Biteen, D. Pacifici, N. S. Lewis, and H. A. Atwater, "Enhanced radiative emission rate and quantum efficiency in coupled silicon nanocrystal-nanostructured gold emitters," Nano Letters, vol. 5, no. 9, pp. 1768-1773, 2005.

[29] J. B. Johnson and R. C. Burt, "The passage of hydrogen through quartz glass," Journal of Optical Society of America, vol. 6, no. 7, pp. 734-738, 1922.

[30] R. M. Barrer, "The mechanism of activated diffusion through silica glass," Journal of the Chemical Society, vol. 136, pp. 378386, 1934. 

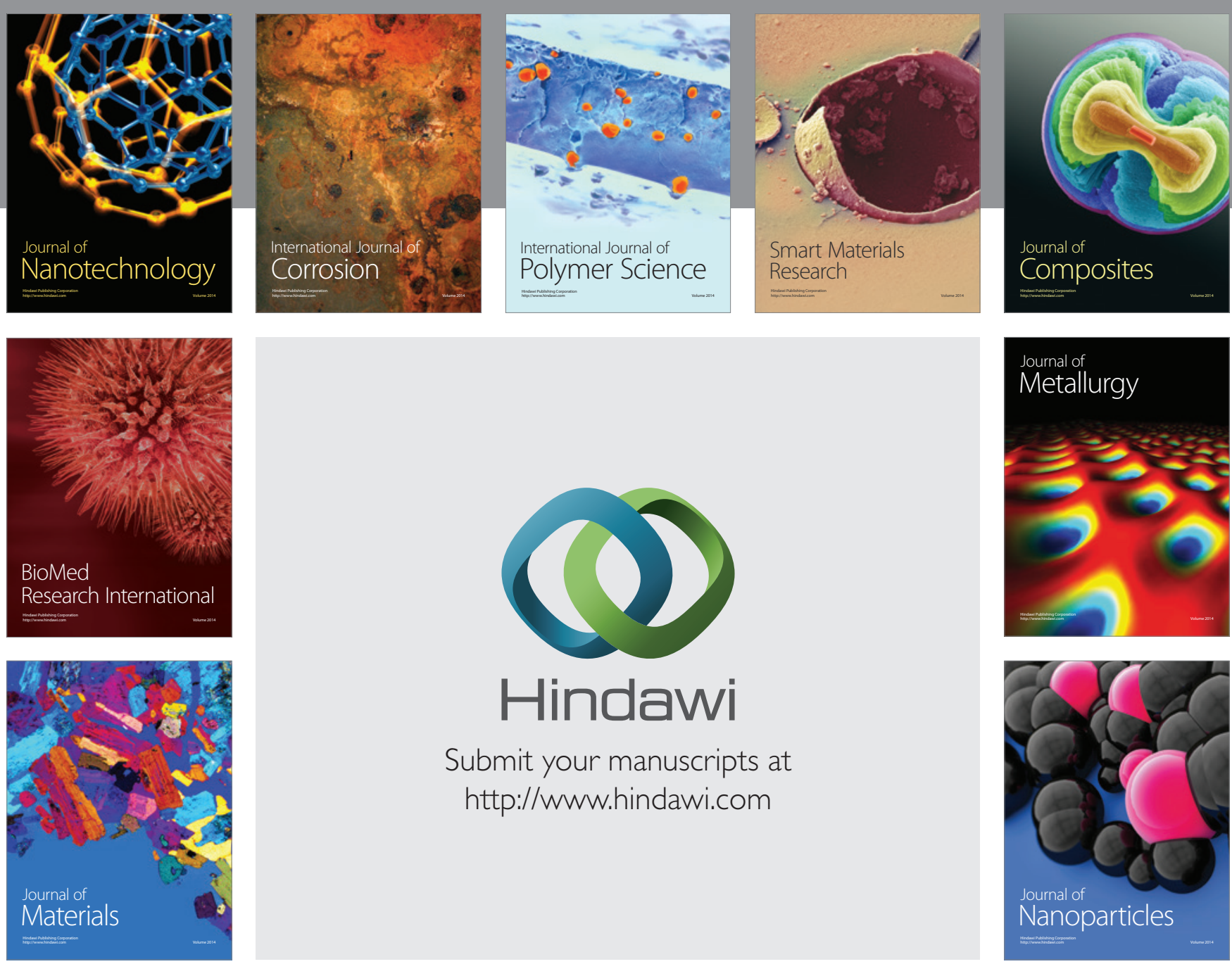

Submit your manuscripts at http://www.hindawi.com
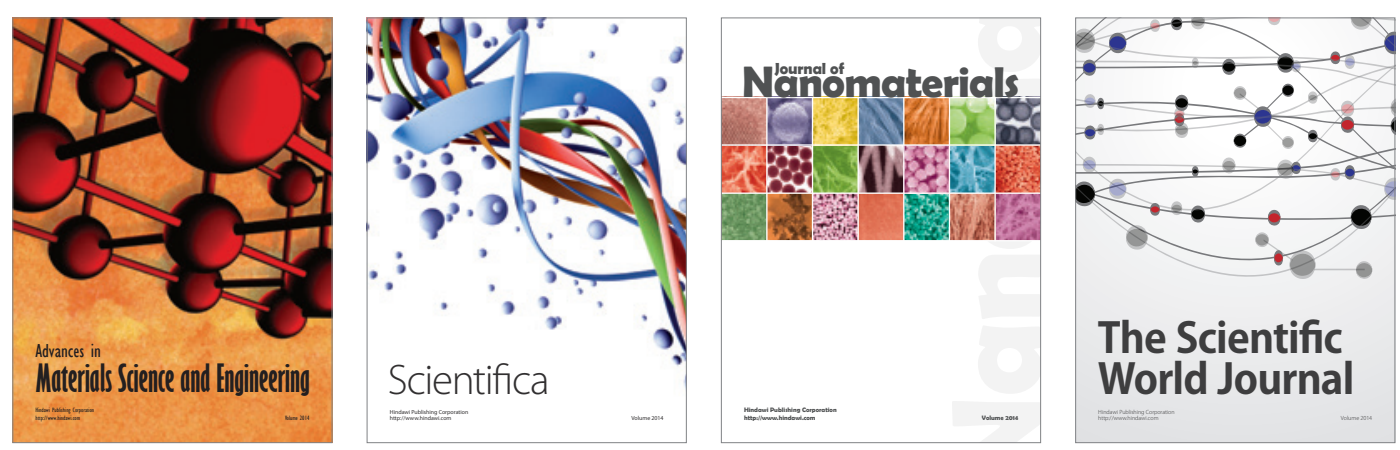

\section{The Scientific World Journal}
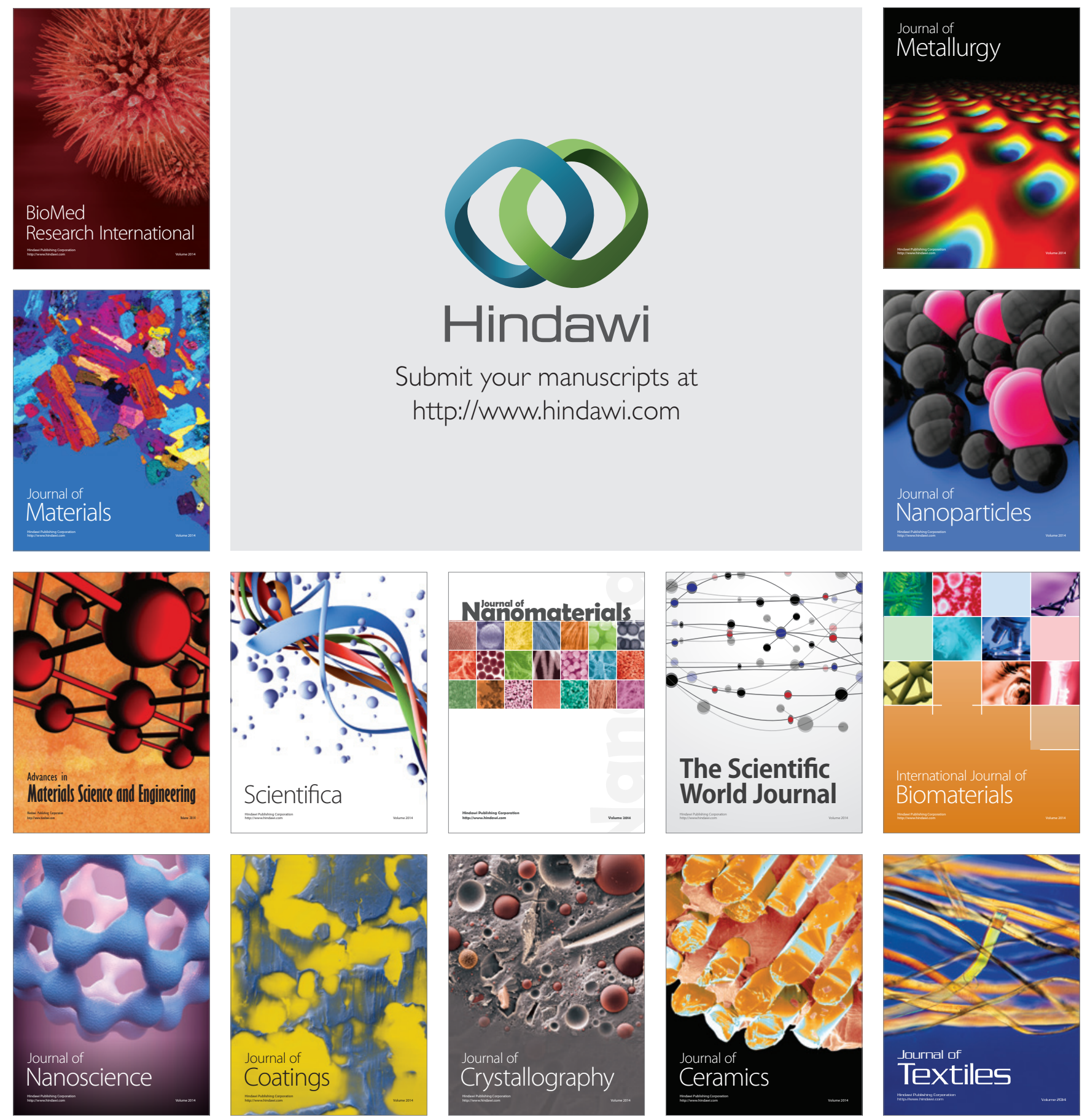